\title{
G. BOYS-STONES, D. EL MURR, Chr. GILL (éd.), The Platonic Art of Philosophy
}

\section{Sylvain Delcomminette}

\section{OpenEdition}

\section{Journals}

Édition électronique

URL : https://journals.openedition.org/philosant/840

DOI : 10.4000/philosant.840

ISSN : 2648-2789

\section{Éditeur}

Éditions Vrin

\section{Édition imprimée}

Date de publication : 1 novembre 2014

Pagination : 331-334

ISBN : 978-2-7574-0855-1

ISSN : $1634-4561$

\section{Référence électronique}

Sylvain Delcomminette, « G. Boys-stones, D. el murR, Chr. GILL (éd.), The Platonic Art of Philosophy », Philosophie antique [En ligne], 14 | 2014, mis en ligne le 01 novembre 2018, consulté le 03 décembre 2022. URL : http://journals.openedition.org/philosant/840 ; DOI : https://doi.org/10.4000/philosant 840

\section{(c)}

Creative Commons - Attribution - Pas d'Utilisation Commerciale - Pas de Modification 4.0 International - CC BY-NC-ND 4.0

https://creativecommons.org/licenses/by-nc-nd/4.0/ 
La neuvième étude, « Myth and eschatology in the Laws », par R. Stalley, est consacrée au mythe du livre $\mathrm{X}$ des Lois et aux différences que son contenu eschatologique présente par rapport aux autres mythes de ce type dans les dialogues précédents - notamment en qui concerne les références au jugement et au châtiment. Contrairement à l'opinion répandue par T. J. Saunders, qui explique ces différences par une évolution de la doctrine eschatologique de Platon vers plus de « scientificité », Stalley soutient, dans une approche pragmatique, que ces différences répondent aux différents rôles joués par les mythes eschatologiques dans les dialogues. Plus précisément, une eschatologie traditionnelle serait contre-productive dans le contexte des Lois, où Platon cherche à présenter une idée de la justice après la mort, plutôt qu'à convertir les citoyens à la philosophie. La question reste ouverte.

Enfin, la dixième étude, «Platonic myth in Renaissance iconography », par E. McGrath, se détache des précédentes par son thème. Il s'agit d'un essai iconologique sur les représentations des mythes platoniciens à la Renaissance. Les thèmes privilégiés par les artistes sont: l'Androgyne du Banquet, le Char Ailé du Phèdre, et la Caverne, dont le potentiel allégorique est exploité dans la mouvance humaniste de Ficin. À partir des exemples choisis, l'étude de McGrath montre efficacement que les figurations des mythes platoniciens à la Renaissance ne sont pas de simples illustrations des textes, mais constituent des interprétations à part entière.

Par la diversité des thèmes traités et par la qualité des travaux présentés, ce volume constitue désormais un outil de travail indispensable pour les spécialistes de Platon et plus généralement pour toute personne intéressée par le rapport complexe que la philosophie entretient, dès ses débuts, avec le mythe.

Leopoldo IRIBARREN

G. Boys-Stones, D. El Murr, Chr. Gill (éd.), The Platonic Art of Philosophy, Cambridge, Cambridge University Press, 2013, x + 341 p. ISBN 978-1-10703898-1.

Cet ouvrage est un recueil d'études rassemblées en l'honneur de Christopher Rowe et consacrées à différents aspects des dialogues de Platon par quelques-uns des spécialistes les plus importants de ce philosophe.

Dans ses très nombreux travaux consacrés à Platon, dont la liste impressionnante figure en fin de volume (p. 312-324), Christopher Rowe a développé une approche originale et extrêmement féconde des Dialogues, qui tranche avec ce que l'on appelait encore il y a quelques années une lecture strictement « analytique » de ce philosophe. Plusieurs caractéristiques de ce dernier type de lecture se trouvent d'une certaine manière illustrées par l'article de T. Penner dans le présent volume («The wax tablet, logic and Protagoreanism », p. 186-220): extraire quelques phrases du texte de Platon, les isoler de leur contexte, les reformuler sous forme de thèses désignées par des sigles, puis les discuter en les confrontant en particulier aux thèses de Frege. Une telle approche fait totalement abstraction de la forme dialoguée des œuvres de Platon ainsi que de leurs aspects «littéraires », qu'elle tient pour dénués de pertinence philosophique. Chr. Rowe, qui, comme un 
certain nombre d'auteurs de ce recueil, a étudié dans un contexte analytique, a au contraire montré que la prise en compte de ces aspects, loin de nous faire quitter le terrain de la philosophie, est essentielle à la compréhension de la pensée platonicienne dans toute sa complexité. C'est la fécondité de ce type de démarche que les auteurs de ce volume cherchent à mettre en évidence par l'étude de problèmes particuliers d'interprétation des Dialogues. Même si leurs résultats divergent parfois, de manière inévitable et salutaire, aussi bien entre eux que de ceux de Chr. Rowe, ce projet commun confère au volume une réelle unité, qui permet de dessiner les contours d'une méthode exégétique dont je m'attacherai ici à dégager quelques lignes de force. (On trouvera un résumé des contributions dans l'Introduction de G. Boys-Stones, p. 3-9.)

Un premier trait commun à plusieurs études réunies ici est l'insistance sur l'importance du contexte général du dialogue étudié pour sa compréhension. Ainsi, U.Zilioli («The wooden horse : the Cyrenaics in the Theaetetus», p. 167-185) cherche à montrer que le Théétète peut être lu comme une réponse de Platon à divers penseurs de son époque, en particulier aux cyrénaïques, qui se cacheraient derrière les kompsoteroi de 156 a et dont la prise en considération permettrait d'éclairer la métaphore du « cheval de bois » en $184 \mathrm{~d}$, mais également à Protagoras et aux autres socratiques. M. Narcy («Why was the Theaetetus written by Euclides ? », p. 150-166) développe plus précisément la piste des Mégariques, en essayant de donner un sens au fait que Platon place l'ensemble du Théétète dans la bouche d'Euclide. S. Broadie («Truth and story in the Timaeus-Critias », p. 249269) et M. Tulli («The Atlantis poem in the Timaeus-Critias », p. 269-282) s'interrogent tous deux sur les raisons qui ont pu pousser Platon à faire de Critias le narrateur du mythe de l'Atlantide dans le Timée et le Critias, mythe que sa famille (qui est aussi celle de Platon) aurait reçu par une transmission secrète en droite ligne de Solon, et y apportent des réponses contrastées mais également stimulantes. Quant à N. Notomi (« Socrates in the Phaedo», p. 51-69), il rappelle notamment que Phlionte, ville où Phédon fait son récit de la mort de Socrate à Échécrate, était intimement liée à Pythagore, qui est lui-même - à tort ou à raison - associé à la naissance du terme «philosophe », ce qui n'est peut-être pas un hasard dans un dialogue où la définition du philosophe joue un rôle central.

Ces remarques montrent déjà combien la composante «dialogique » est essentielle à la pensée de Platon. À la surface, il y a le dialogue qui se déroule entre les interlocuteurs de l'œuvre écrite. Mais ce premier niveau peut cacher un dialogue plus profond entre Platon et d'autres penseurs, qu'ils lui soient antérieurs ou contemporains. Ce dialogue peut également se nouer par des liens d'intertextualité, comme le montre M. Schofield («Friendship and justice in the Laws», p. 283297) à propos de la signification et du rôle politiques de l'amitié dans les Lois, où il décèle une manœuvre littéraire et historiographique complexe dans une référence à des vers prêtés à Lycurgue. À un niveau de complexité supérieur encore, cette intertextualité peut également être imaginaire : ainsi, lorsque Platon invente un poème de Solon dont il prétend s'inspirer dans le récit atlante du Timée et du Critias, comme le souligne M. Tulli. Bien plus, par l'exploitation de diverses ressources dramatiques et rhétoriques, Platon instaure un dialogue avec son lecteur en le forçant à adopter une attitude active dans la lecture et à penser par lui-même, comme le 
met en évidence le texte de M. Dixsaut qui ouvre le recueil (« Macrology and digression $\gg$, p. 10-26). Cette dimension dialogique de la pensée est bien mise en œuvre dans ce recueil au niveau de l'exégèse elle-même, non seulement par les nombreuses discussions des interprétations de Chr. Rowe qui parcourent l'ouvrage, mais également par l'excellente idée des éditeurs scientifiques d'insérer deux «Commentaires réflexifs » (l'un par Chr. Gill, «'Socratic' psychology in Plato's Republic », p. 110-121, et l'autre par D. El Murr, «Appearance, reality and the desire for the good », p. 122-129) qui reviennent sur la manière dont d'autres textes du recueil (en particulier ceux de D. Sedley et de T. Johansen) se sont positionnés par rapport à celles-ci. On peut également mentionner ici la discussion serrée proposée par D. O'Brien («A form that 'is' of what 'is not' : existential einai in Plato's Sophist», p. 221-248) des diverses interprétations (essentiellement anglo-saxonnes) de l'être et du non-être dans le Sophiste, bien qu'elle soit plus polémique et sans lien direct avec les travaux de Chr. Rowe.

La prise en compte de ces différents éléments permet également d'affirmer la grande unité de chaque dialogue, même là où celle-ci a été contestée. Ainsi, N. Notomi montre qu'il n'y a pas lieu de distinguer les aspects «socratiques » des aspects « platoniciens » dans un dialogue comme le Phédon, qui s'articule tout entier autour de la question du philosophe tel qu'il est déjà décrit dans l'Apologie. D. Sedley («Socratic intellectualism in the Republic's central digression », p. 7089) argumente en faveur de la division de la République en six livres plutôt que dix, dont nous savons qu'elle a existé dans l'Antiquité, division qui rendrait le dialogue plus unitaire en évitant les articulations trop tranchées, et cherche à montrer sur cette base que l'apparente opposition que l'on pourrait soupçonner entre la psychologie tripartite développée dans ce que nous appelons les livres IV, VIII et IX et la psychologie unitaire du Phédon qui semble gouverner toute la digression centrale des actuels livres V à VII s'explique par les objectifs différents de ces deux types d'explications. M. M. McCabe («Waving or drowning ? Socrates and the sophists on self-knowledge in the Euthydemus », p. 130-149) s'attache à manifester les connexions complexes entre les différents niveaux de l'Euthydème - le dialogue avec Criton qui l'encadre, la succession des sophismes, les épisodes « socratiques » qui interrompent cette dernière - autour d'un débat sur la nature de la connaissance.

Mais la lecture de Chr. Rowe n'est pas seulement unitariste relativement à chaque dialogue pris séparément : elle s'écarte également de manière décisive de toute tentation « développementaliste », et en particulier de celle qui chercherait à distinguer une période «socratique » d'une période proprement «platonicienne » dans son œuvre. Cette question est poursuivie par plusieurs contributions du présent volume. N. Notomi, D. Sedley et Chr. Gill nient tous trois la pertinence d'une distinction tranchée entre ce qui serait «socratique » et ce qui serait « platonicien », quoique à des titres divers. Selon D. Sedley, nous devons partir du fait que Platon lui-même avait une vision « unitariste » de son propre développement. Chr. Gill suggère de son côté que cette question n'intéressait tout simplement pas Platon, à tel point qu'au lieu de nuancer la distinction entre « socratique » et « platonicien », il conviendrait de l'abandonner totalement lorsque nous cherchons à interpréter Platon. À propos du problème particulier constitué par les traitements apparemment divergents du corps humain dans les Dialogues, 
celui-ci apparaissant tantôt comme un obstacle à la connaissance et tantôt comme un véhicule de l'âme nous permettant de nous élever vers le divin, M. A. Fierro (« Two conceptions of the body in Plato's Phaedrus », p. 27-50) propose une explication fondée sur la différence entre les projets et les objectifs dialectiques de chaque dialogue et souligne que ces différentes approches peuvent également être combinées au sein d'un dialogue unique, ce qui se produit dans le Phèdre. Quant à T. Johansen («Timaeus in the cave», p. 90-109), il met au jour les connexions profondes entre la République et le Timée, la première, selon la formule des anciens commentateurs, disant « de manière éthique » ce que le second dit « de manière physique », chacun des deux aspects étant néanmoins présent dans les deux dialogues, manifestant ainsi les connexions essentielles entre la sphère humaine et le reste du cosmos.

Ces quelques remarques ne visent aucunement à dresser le portrait complet d'une méthode unitaire qui serait partagée par l'ensemble des contributeurs de ce volume. Elles cherchent seulement à suggérer la fécondité du type d'approche dont Chr. Rowe est l'un des défenseurs les plus actifs et les plus éminents, et par conséquent la richesse de ce très beau volume qui lui est offert, dont les contributions de haut niveau ne manqueront pas de stimuler la réflexion de tous les amoureux de Platon.

Sylvain DELCOMMINETTE Université Libre de Bruxelles

Dimitri El MURR (éd.), La Mesure du Savoir: études sur le Théétète de Platon, Paris, Librairie philosophique J. Vrin, 2013 (Tradition de la pensée classique), 426 pages, ISBN 978-2-7116-2495-9.

La première étude de ce substantiel recueil de travaux savants sur le Théétète est signée de Marc-Antoine Gavray. L'objectif est de faire apparaître, à l'occasion de la citation du livre La Vérité de Protagoras, la leçon d'herméneutique qui se met en place, excédant le simple cadre de la réfutation. Lire en philosophe, ce n'est pas simplement expliquer, analyser, saisir les présupposés de la thèse, mais la comprendre: «L'herméneutique platonicienne se présente (...) comme la recherche d'une collaboration en vue d'un cheminement commun qui postule la diffusion de l'écrit, la notoriété de son auteur, l'intérêt de son contenu et la possibilité de l'accompagner dans le raisonnement (...) » (p. 28). La formule est intéressante mais peut porter à confusion, car M.-A. Gavray prend la diffusion du livre comme une donnée allant de soi, non problématique à la base. Dans le Théétète, la référence au livre La Vérité (162a) n'est effective que parce qu'elle est replacée dans le contexte de l'examen du «nouveau-né » de Théétète, précisément au cours de la procédure de l'amphidromie comme «promenade» du rejeton (160e-161a). En conséquence, ce qui est examiné c'est moins le livre de Protagoras que le fruit qui en résulte chez un lecteur particulier, en l'occurrence Théétète. Car, bien évidemment, le jeune homme a été ensemencé par sa lecture. Malheureusement, la caractéristique spermatique du «fragment» (p.28) n'a pas été relevée, alors que la courte citation de l'homme-mesure a pu produire tout ses effets... Il est pourtant question de sperma dans le discours maïeutique du Théétète (149e4). Il en résulte 